\title{
Article
}

\section{Forecasting the space weather impact: The COMESEP project}

Crosby, N. B., Veronig, A., Robbrecht, E., Vrsnak, B., Vennerstrom, S., Malandraki, O., Dalla, Silvia, Rodriguez, L., Srivastava, N., Hesse, M., Odstrcil, D. and COMESEP, Consortium

Available at https://clok.uclan.ac.uk/28203/

Crosby, N. B., Veronig, A., Robbrecht, E., Vrsnak, B., Vennerstrom, S., Malandraki, O., Dalla, Silvia orcid iconORCID: 0000-0002-7837-5780, Rodriguez, L., Srivastava, N. et al (2012) Forecasting the space weather impact: The COMESEP project. AIP Conference Proceedings, 1500 (1). pp. 159164. ISSN 0094-243X

It is advisable to refer to the publisher's version if you intend to cite from the work. http://dx.doi.org/10.1063/1.4768760

For more information about UCLan's research in this area go to http://www.uclan.ac.uk/researchgroups/ and search for <name of research Group>.

For information about Research generally at UCLan please go to http://www.uclan.ac.uk/research/

All outputs in CLoK are protected by Intellectual Property Rights law, including Copyright law. Copyright, IPR and Moral Rights for the works on this site are retained by the individual authors and/or other copyright owners. Terms and conditions for use of this material are defined in the policies page.

\section{CLoK}

Central Lancashire online Knowledge www.clok.uclan.ac.uk

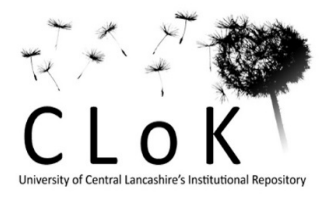




\section{AIP Conference Proceedings}

Forecasting the space weather impact: The COMESEP project

N. B. Crosby, A. Veronig, E. Robbrecht, B. Vrsnak, S. Vennerstrom et al.

Citation: AIP Conf. Proc. 1500, 159 (2012); doi: 10.1063/1.4768760

View online: http://dx.doi.org/10.1063/1.4768760

View Table of Contents: http://proceedings.aip.org/dbt/dbt.jsp?KEY=APCPCS\&Volume=1500\&lssue=1

Published by the American Institute of Physics.

\section{Related Articles}

Ring current instabilities excited by the energetic oxygen ions

Phys. Plasmas 14, 092902 (2007)

Quasielectrostatic instabilities excited by energetic oxygen ions in the ring current region

Phys. Plasmas 12, 012903 (2005)

Charge exchange imaging of space plasmas (invited)

Rev. Sci. Instrum. 75, 3526 (2004)

On nonlinear plasma instabilities during the substorm expansive phase onset

Phys. Plasmas 11, 2046 (2004)

Interpretation of Cluster data on chorus emissions using the backward wave oscillator model

Phys. Plasmas 11, 1345 (2004)

\section{Additional information on AIP Conf. Proc.}

Journal Homepage: http://proceedings.aip.org/

Journal Information: http://proceedings.aip.org/about/about_the_proceedings

Top downloads: http://proceedings.aip.org/dbt/most_downloaded.jsp?KEY=APCPCS

Information for Authors: http://proceedings.aip.org/authors/information_for_authors

\section{ADVERTISEMENT}

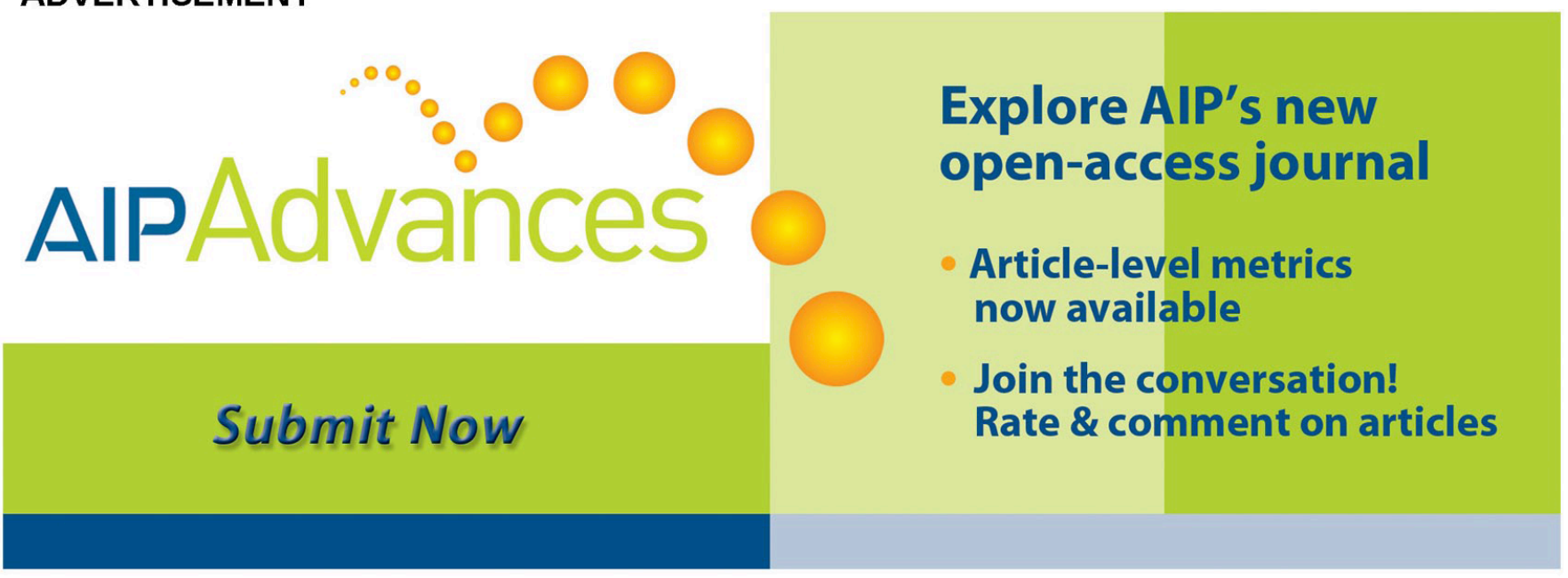




\title{
Forecasting the Space Weather Impact: the COMESEP Project
}

\author{
Crosby, N.B. ${ }^{\text {a; }}$ Veronig, A. ${ }^{\text {b }}$ Robbrecht, E. ${ }^{\text {c; }}$ Vrsnak, B. ${ }^{\text {d }}$; \\ Vennerstrom, S. ${ }^{\text {e }}$ Malandraki, O. ${ }^{\text {f }}$; Dalla, S. ${ }^{\mathrm{g}}$; Rodriguez, L. ${ }^{\mathrm{c}}$; \\ Srivastava, N. ${ }^{\mathrm{h}}$; Hesse, M. ${ }^{\mathrm{i}}$; Odstrcil, D. ${ }^{\mathrm{i} j}$; on behalf of the COMESEP \\ Consortium
}

\begin{abstract}
${ }^{a}$ BIRA-IASB, Belgium; ${ }^{b}$ University of Graz, Austria; ${ }^{c}$ Royal Observatory of Belgium, Belgium; ${ }^{d}$ Hvar Observatory, Croatia; ${ }^{e}$ Technical University of Denmark, Denmark; ${ }^{f}$ National Observatory of Athens, Greece; ${ }^{g}$ University of Central Lancashire, UK; ${ }^{h}$ Udaipur Solar Observatory, India;

${ }^{i}$ CCMC, NASA Goddard Space Flight Center, USA; ${ }^{j}$ George Mason University, Fairfax.
\end{abstract}

\begin{abstract}
The FP7 COronal Mass Ejections and Solar Energetic Particles (COMESEP) project is developing tools for forecasting geomagnetic storms and solar energetic particle (SEP) radiation storms. By analysis of historical data, complemented by the extensive data coverage of solar cycle 23, the key ingredients that lead to magnetic storm and SEP events and the factors that are responsible for false alarms are being identified. To enhance our understanding of the 3-D kinematics and interplanetary propagation of coronal mass ejections (CMEs), the structure, propagation and evolution of CMEs are being investigated. In parallel, the sources and propagation of SEPs are being examined and modelled. COMESEP is a unique cross-collaboration effort and bridges the gap between the SEP, CME and terrestrial effects scientific communities.
\end{abstract}

Keywords: Space Weather Forecasting; Coronal Mass Ejections; Solar Energetic Particles PACS: 94.05.Sd; 96.60.ph; 96.50.Vg.

\section{INTRODUCTION}

The COMESEP (COronal Mass Ejections and Solar Energetic Particles: forecasting the space weather impact) Project (http://www.comesep.eu) is developing tools for forecasting geomagnetic storms and SEP radiation storms based on extensive scientific data analysis, model development and risk analysis. COMESEP is funded by the European Union Framework Programme 7 (FP7) and is a three-year collaborative project that kicked off on 1 Feb. 2011. COMESEP is a unique cross-collaboration effort and bridges the gap between the SEP, CME and terrestrial effects scientific communities. Its overall objective is to build an operational space weather alert system to forecast SEP radiation storms and geomagnetic storms, and to perform risk analysis for selected user groups.

COMESEP complements the ESA Space Situational Awareness (SSA) activities for security of space assets from space weather events. It is providing a new European service for vulnerable technologies both in space and on the ground. While many forecasting projects end at the model stage, COMESEP will go one step further by quantifying model outputs in terms of magnetic storm and SEP radiation storm levels.

This paper reviews the individual objectives of the COMESEP project and presents the main outcomes of the first year. 


\section{BASIC SCIENCE}

The basic science that is being pursued in the COMESEP Project is two-sided. It includes data analysis (real-time data and historical data) as well as modelling of both SEP events and CME propagation.

\section{Coronal Mass Ejections}

In regard to CMEs the Project is pursuing several main objectives. They include: 1.) Enhancing our understanding of the three-dimensional (3-D) propagation of interplanetary CMEs (ICMEs), by combining in situ measurements from a number of space probes at various heliospheric locations with remote (coronagraphic) observations, 2.) Testing and comparison of the analytical and numerical ICME propagation models, including improvement of their performances and identification of the model-input parameters that have largest effect on the model output, 3.) Investigating the evolution of the ICME magnetic structure inferred from the in situ measurements at various locations in the heliosphere, including implications for predicting geoeffectiveness. In summary, the main goals are to advance the understanding of the 3-D propagation of ICMEs, which is needed to improve and optimise the performances of ICME propagation models, as well as to enhance our comprehension of the ICME magnetic field structure and its evolution, required for predicting geoeffectiveness. Both aspects are essential for developing the COMESEP space weather alert system and represent the building blocks for the work that is being performed in the Project.

A new technique was developed (Rollett et al., 2012) for deriving the propagation direction and kinematics of ICMEs, based on combining the remote sensing data from STEREO Heliospheric Imagers (HI) and 1 AU in situ measurements (arrival time and impact speed). Supplementing this method with another newly developed "self-similar expansion method" (Möstl and Davies, 2012), the propagation direction and velocity evolution of a number of well observed ICMEs was derived over the entire Sun-Earth range. This latter new method not only traces the ICME nose kinematics but also the flank kinematics.

Most of the analysed ICMEs were used to test and optimise the Drag-Based Model (DBM; Vrsnak et al., 2012). The study included analysis of ICME-ICME interactions and variable ambient solar wind speed. The DBM was made public as an online tool (http://oh.geof.unizg.hr/CADBM/cadbm.php), and has been used on several occasions for real-time forecasting of ICME arrivals through the "SOHOalert" system. For this purpose, a statistical study of several CME/ICME samples was performed to find the range of values of the DBM-input parameters. It was established that the "drag-parameter" $\gamma$ is generally in the range $210^{-8}-210^{-7} \mathrm{~km}^{-1}$, whereas the solar wind speed $w$ ranges between $300-700 \mathrm{~km} / \mathrm{s}$. For a given ICME take-off speed, such a span of input values can lead in most extreme cases, to a difference in the predicted transit time as large as 50\%, however, typically it is around 20\% (Vrsnak et al. 2012; see also Vrsnak et al 2007, 2010). In the case of very fast CMEs the $20 \%$ uncertainty corresponds to a transit time difference of several hours, whereas in the case of very slow CMEs it can be as large as 1 day. In a statistical sense, the optimum values turn out to be $\gamma=10^{-7} \mathrm{~km}^{-1}$ and $w=500$ $\mathrm{km} / \mathrm{s}$, so these values are set as default input parameters in the DBM-online tool. In real-time forecasting it was found that the transit-time error is typically better than 12 hours. The DBM calculation takes a few seconds. Therefore any time delay 
depends primarily on the coronagraphic data acquisition rate. The geoeffectiveness of a CME is mainly given by $B_{z}$ and the speed of the ICME. $B_{z}$ is very difficult to determine from solar observations and there are indications that CMEs may rotate during their travel to the Earth, making any early estimation of $\mathrm{B}_{\mathrm{z}}$ problematic. Here we aim to estimate the ICME arrival times and to identify the key solar parameters of importance for space weather impact. The model prediction of $B_{z}$ is not attempted in this work.

\section{Solar Energetic Particles}

In regard to SEPs the main objectives of the Project include enhancing our understanding of sources, acceleration processes, as well as the propagation of SEPs, by means of data analysis and modelling. Advanced SEP modelling to include CME propagation effects, cross-field diffusion and heavy ions is being investigated. During the first year a literature survey of previous space weather effective SEP event lists has been performed. This has resulted in the compilation of an SEP event list based on the SEPEM proton reference channel (7.23 - 10.45 $\mathrm{MeV}$ ) (http://dev.sepem.oma.be/) and associated solar events.

Two SEP events in Nov. 2001 exhibiting 'reservoir' characteristics were identified by having near-equal intensities and statistically significant heavy ion measurements at ACE and Ulysses. A study of the compositional and spectral comparisons during these reservoir periods is in progress. Ulysses and ACE have measured statistically significant heavy ion flux values for these events and thus serve well for studying the evolution of SEP characteristics at two different locations in the 3-D heliosphere. It has been shown in the past that the perpendicular to parallel diffusion coefficient ratio could be as large as unity with some prescribed magnetic turbulence (e.g. Giacalone and Jokipii 1999). During the decay phase of the SEP events, in the downstream regions of shocks which are known to be characterised by enhanced magnetic turbulence, cross-field diffusion operates enabling particles to occupy different flux tubes which provides a possible explanation for the remarkable energetic particle spectral invariance observed.

Furthermore, using the intensity characteristics and the angular distributions of both incident and reflected particle beams, two large SEP events detected on 2 May 1998 and 21 April 2002 during the passage of magnetic clouds were identified with evidence of reflecting boundaries ahead of the spacecraft in space. The onset time analysis of non-relativistic electrons and $\sim \mathrm{MeVnucleon}^{-1}$ heavy ions was performed and exhibits the stability of the magnetic loop structure during a period of a few hours in the events examined. The joint analysis of pitch-angle distributions (PADs) and peak intensities of electrons indicates that in the April event the reflected particles with nearly zero pitch-angle at 1 AU could reach the vicinity of the Sun, implying that the magnetic loop was a magnetic bottle connected to the Sun with both legs. In contrast, in the May event the magnetic mirror was formed by a compressed field enhancement behind the interplanetary shock driven by a preceding CME, being consistent with its open field line topology. Analysis of the decay phase was performed for the two events and the implications for the particle reservoir formation were examined. To this goal, the spatial and temporal variation of the high-energy proton intensities in the inner heliosphere is examined, through the investigation of particle confinement mechanisms occurring in individual flux tubes in order to understand the 
conditions that enhance the duration of high-energy proton intensities in different magnetic topologies (Tan et al., 2012).

Investigation into SEP sources, and acceleration processes operating on the SEPs in terms of solar flares, ICMEs and their associated shocks has been carried out. During the period 1997-2006 two SEP events occurring in Dec. and Aug. 2001 were identified as having an initial ratio of $\mathrm{Fe} / \mathrm{O}>0.8$, constructed from the provided $\mathrm{Fe}$ and $\mathrm{O}$ intensities at $\sim 5 \mathrm{MeV} /$ nucleon. They were selected for a study concerning the transport and acceleration of SEPs in the low solar corona. Ulysses/COSPIN/LET and WIND/EPACT/LEMT data were used for this investigation. During the two events transient $\mathrm{Fe} / \mathrm{O}$ enhancements were seen at both WIND at L1 and Ulysses although one or both spacecraft were not 'wellconnected' to the flare. The observations demonstrate that initial $\mathrm{Fe} / \mathrm{O}$ enhancements cannot be cited as evidence for a direct solar flare component but rather they can be better understood as a transport effect, driven by the different mass-to-charge ratios of Fe and $\mathrm{O}$ (Tylka et al., 2012)

Scientific results obtained serve as observational constraints on the SEP propagation model. A test particle code for the study of SEP propagation from the Sun to near-Earth regions has been developed. Two main interplanetary magnetic field configurations have been analysed: a Parker spiral configuration and one based on a model of large-scale heliospheric turbulence by Giacalone (1999). A scheme for scattering was implemented within the test particle model: each particle's velocity is scattered by a random angle with an average frequency determined by the value of the mean free path $\lambda$. To characterise the transport of SEPs across the field a local 'Parker spiral' coordinate system was introduced and coordinate transformations for the determination of transport coefficients were derived. The ratio of the parallel to perpendicular diffusion coefficient can then be obtained as an output of the simulation. A low-scattering regime with $\lambda=2$ AU was compared with a high-scattering regime with $\lambda=0.3 \mathrm{AU}$, and with the scatter-free case. It was found that transport across the magnetic field is enhanced in the $\lambda=2 \mathrm{AU}$ and $\lambda=0.3 \mathrm{AU}$ cases, compared to the scatter-free case. Values of the ratios of perpendicular to parallel diffusion coefficient vary between 0.01 and 0.08 (Kelly et al., 2012). The simulations also showed that transport across the field in heliolatitude may be less efficient than in heliolongitude.

\section{Space Weather Impact}

This part of the Project concerns the impact that the space weather has on the near-Earth space environment. Two types of space weather impact are being investigated during the SOHO era: the occurrence of large geomagnetic storms and significant SEP events. For this purpose a comprehensive database of event data is being constructed; this includes information about all the key observable parameters as well as the associated geomagnetic and SEP impacts observed near Earth.

The data collected will serve as input for a statistical analysis addressing the question what is the probability that a given solar event will produce a major space weather event. The impact of particular parameters and their mutual relationship will be investigated. Furthermore, the potential increase in the probability of large space weather impacts when multiple events occur will also be studied. All the obtained probabilities will be folded in the risk estimates. It is essential that the information on the parameters considered above is available in near-real time for 
useful predictive purposes. Only a few large events exist in the SOHO time period and for this reason very large events from the pre-SOHO / pre-space era will also be investigated.

One of the main objectives of the Project is to identify the major causes of false alarms following solar observations and thereby create the basis for suggesting possible solutions to the problem. In COMESEP a false alarm is defined as a solar event with characteristics that would lead one to expect either a magnetic storm and/or an SEP event, but which does not actually produce one. The first part of this task was to identify a list of potential geo-effective solar events which did not cause significant space weather effects (e.g. the event did not hit the Earth/ the event did not contain significant southward IMF for geomagnetic storms, no or poor magnetic connection to source region for SEPs).

\section{DEVELOPMENT OF FORECASTING METHODS}

To develop the various forecasting methods the models describing the propagation of CMEs and SEPs for space weather forecasting will be optimised and built upon the scientific understanding obtained in Section 2. An automated CME detection system will be implemented and the Project is obtaining information on the background solar wind parameters from several solar wind models. The Project will link the CME detection system and solar wind modelling with the CME and SEP forecasting tools so that key parameters from the former are passed to the tools.

The first component of the COMESEP pipeline is an automated system capable of detecting CMEs and determining the key parameters. This is CACTus (http://sidc.be/cactus) which autonomously detects CMEs in image sequences from $\mathrm{SOHO} / \mathrm{LASCO}$ in near-real time (Robbrecht and Berghmans, 2004). This tool will be used in conjunction with NEMO (Novel EIT wave Machine Observing, http://sidc.be/nemo, Podladchikova and Berghmans, 2005) to identify frontside CMEs.

The ICME propagation model being developed within the COMESEP Project requires information on the background solar wind as input. Two models, [ENLIL/MAS (MHD-Around-A-Sphere); Linker et al., 1999; Mikic et al., 1999; Riley et al., 2001] and [ENLIL/WSA (Wang-Sheeley-Arge); Arge and Pizzo, 2000] were used. Both models were found to reproduce general features of background solar wind and recurrent structures. However predicted arrival times of high speed solar wind streams have uncertainties of about $1-1.5$ days. In addition, an empirical method that predicts solar wind conditions based on a fully automatic algorithm for identifying coronal holes in SOHO EIT 195 Á images (Rotter et al., 2012) was assessed. Using this latter method the best results were obtained for predictions of solar wind speed.

As part of the work a preliminary version of an online tool for forecasting solar wind characteristics and geomagnetic activity associated with co-rotating interaction regions has been made available at http://oh.geof.unizg.hr/CH/ch.php.

\section{ALERT SYSTEM}

A main objective of the Project is to create a system alerting about potential harmful space weather events, to provide an automated integrated start-to-end 
service, and to disseminate alerts to space weather vulnerable industries. The system will work as a patchwork of building blocks distributed on several nodes that interact with each other. The system will be event driven and can communicate alerts to the end users.

In addition to the forecasting methods described above, a number of additional tools are also being considered for development and inclusion in the COMESEP alert system. These tools aim at easing the tasks of forecasting ICME arrival times and estimating the risk of SEP events.

Once all the building blocks are set together and working, the system needs to deal with the risk analysis for geomagnetic and SEP radiation storms. The work planned here consists of identifying different types of users that regularly experience the effects from SEP radiation storms (e.g. human exploration, satellite operators) and geomagnetic storms (e.g. power companies, global positioning system users, satellite operators), and perform a risk-analysis for these users. In general, a risk matrix will be implemented. Considering the magnitude of the event vs. the likelihood of occurrence, the output of this system will provide low, medium, high and extreme level risk alerts.

\section{ACKNOWLEDGMENTS}

The authors acknowledge all members of the COMESEP Consortium. This work has received funding from the European Commission FP7 Project COMESEP

(263252).

\section{REFERENCES}

1. Arge, C.N. and Pizzo, V.J., J. Geophys. Res, 105, 10465, 2000.

2. Giacalone, J., Adv. Space Res. 23, 581-590, 1999.

3. Giacalone, J., Jokipii, J. R., Astrophys. J., 520, 204, 1999.

4. Kelly, J., Dalla, S., Laitinen, T., Astrophys. J., 750, 47, 2012.

5. Linker, J.A., Mikic, Z., Biesecker, D.A., et al., J. Geophys. Res., 104, 9809, 1999.

6. Mikic, Z., Linker, J. A., Schnack, D. D., Lionello, R., Tarditi, A., Phys. Plasmas, 6, 2217, 1999.

7. Möstl, C. and Davies, J.A., in Solar Physics Topical Issue "Remote Sensing of the Inner Heliosphere", 2012 (revised).

8. Podladchikova, O. and Berghmans, D., Sol. Phys., 228(1), 267-286, 2005.

9. Riley, P., Linker, J. A., Mikic, Z., J. Geophys. Res., 106, 15889, 2001.

10. Robbrecht, E. and Berghmans, D., A\&A, 425, pp. 1097, 2004.

11. Rollett, T., Möstl, C., Temmer, M., Veronig, A.M., Farrugia, C.J., Biernat, H.K., Solar Phys., 276, Issue 1, 2012.

12. Rotter T., Veronig, A.M., Temmer, M., Vrsnak, B., Solar Physics, 2012 (under review).

13. Tan, L.C., Malandraki, O.E., Reames D. V., Ng, C. K., Wang L., Dorrian, G., Ap J, 750, 146, 2012.

14. Tylka, A.J., Malandraki, O.E., Dorrian, G., Ko, Y.-K., Marsden, R.G., Ng, C.K., Tranquille, C., Solar Physics, published online, July 2012.

15. Vrsnak, B., Zic, T., A\&A, 472, 937, 2007.

16. Vršnak, B., Zic, T., Falkenberg, T. V., Möstl, C., Vennerstrom, S., Vrbanec, D., A\&A, 512, A43, 2007.

17. Vrsnak B., Zic T., Vrbanec, D., Dumbovic, M., Calogovic J., Veronig A.M., Temmer M., Mostl C., Rollett T., Bein B., Lulic S., Moon Y.-J.: Solar Phys., 2012 (published as "online first”, DOI 10.1007/s11207-012-0035-4). 